\title{
LA COMPETENCIA COMUNICATIVA EN ESCOLARES DE EDUCACIÓN PRIMARIA
}

\author{
COMUNICATIVE COMPETENCE IN PRIMARY SCHOOL CHILDREN
}

\author{
Isabel Gómez, Veiga* y Pilar Vieiro** \\ Universidad da Coruña
}

\begin{abstract}
RESUMEN
El trabajo que presentamos en este artículo pretende contribuir al conocimiento de la competencia comunicativa desarrollada en la lengua oral por los escolares de ocho años. Analizamos los discursos producidos en la primera y en la segunda lengua, a través de medidas relacionadas con el acceso al léxico, la precisión sintáctica y la complejidad de las construcciones gramaticales construidas por los hablantes. Los resultados apuntan a que las diferencias en la competencia comunicativa en ambos códigos se relacionan, fundamentalmente, con los procesos básicos de codificación léxica y morfosintáctica.
\end{abstract}

Palabras clave: primera lengua, segunda lengua, competencia comunicativa.

\begin{abstract}
The work reported in this paper purports to investigate communicative competence in oral language developed by school children (average age 8:2). Discourses produced in both first language and second language were analysed through measures related to lexical access, syntactic accuracy and complexity of grammatical structures constructed by speakers. Results indicate that differences in communicative competence between codes are associated with basic processes of lexical and morphosyntactic encoding.
\end{abstract}

Keywords: first language, second language, communicative competence.

* Isabel Gómez Veiga es Becaria predoctoral por la Xunta de Galicia en el Departamento de Psicología Evolutiva y de la Educación de la Universidad de A Coruña. Ha participado en proyectos de investigación acerca de los factores cognitivos y contextuales determinantes de la comprensión y producción del discurso.

** La Dra. Pilar Vieiro es Profesora Titular en la Facultad de Ciencias de la Educación de la Universidad de A Coruña. Actualmente imparte docencia en las titulaciones de Psicopedagogía y Magisterio. Sus trabajos de investigación y publicaciones se centran, por una parte, en la indagación acerca de los procesos cognitivos implicados en la comprensión y la producción del discurso y, por otra, en el desarrollo de programas de intervención para la mejora de la comprensión y el aprendizaje a partir de los textos. 


\section{Introducción}

En los últimos años, se viene proponiendo un cambio en los planteamientos instruccionales de las segundas lenguas, desplazando a los métodos tradicionales, muy estructurales y directivos, a favor del desarrollo de programas más «comunicativos». A partir de los trabajos realizados por los analistas del discurso (entre otros, Chaudron, 1988), ha surgido la consideración del aula como un espacio de intercambio de significados, en el cual tienen cabida, por lo tanto, los presupuestos de una enseñanza comunicativa. Este giro viene avalado por los resultados de la investigación psicolingüística que subrayan la estrecha relación entre el uso de la lengua y el desarrollo de los procesos de adquisición de la misma (Swain, 1985). El planteamiento subyacente es que los alumnos adquieran una segunda lengua (L2, en adelante) a través de procesos de comunicación e interacción semejantes a los que han puesto en marcha cuando adquirieron su primera lengua (L1, en adelante), avanzando desde un manejo instrumental del código lingüístico hasta la reflexión acerca del mismo. En este contexto, el concepto de competencia comunicativa se revela como el motor de cambio de la enseñanza de las lenguas, en general, y de las segundas lenguas, en particular, situada en el centro de un continuum delimitado por la capacidad de uso del lenguaje, en un extremo, y el conocimiento formal del mismo, en el otro (Esteaire y Zanón, 1990).

Si consideramos la capacidad de utilizar la L2, un breve análisis de cualquier producción espontánea nos revela que el hablante dedica gran parte del tiempo y esfuerzo al proceso de negociación del significado a transmitir, así como a la búsqueda de soluciones a los problemas que la comunicación en tiempo real le plantea. Por lo tanto, comprender cómo se pueden gestionar y optimizar los mecanismos o estrategias puestos en marcha en tales circunstancias se convierte en un objetivo relevante en la investigación acerca de la adquisición de un segundo código, tanto por sus implicaciones en el plano teórico, como en el práctico. En el ámbito teórico porque nos permite profundizar en el conocimiento del procesamiento lingüístico en situaciones de déficit de recursos; en el ámbito práctico porque, a pesar de la persistencia de dichos mecanismos en el discurso de los aprendices y de que, en principio, parece posible su enseñanza (Dörnyei, 1995), no siempre los materiales y los proyectos educativos se plantean proporcionar cierto bagaje estratégico que permita solventar potenciales dificultades en el transcurso de la comunicación.

En general, en una situación comunicativa que requiere el uso de la L2, los principales problemas para articular un mensaje acorde con la intención comunicativa del hablante se suelen producir:

a) en el acceso a la unidad léxica planificada,

b) en la construcción del marco sintáctico correcto según el código en uso, y

c) en los procesos de codificación fonológica (Levelt, 1989).

Para superar tales dificultades, los hablantes pueden optar por abandonar o cambiar el mensaje que originalmente han planificado, o bien, pueden decidir modificar sólo algunos elementos del mensaje preverbal (Poulisse, 1994). Tales opciones pueden ser ejecutadas a través de diversas estrategias que el individuo emplea a modo de mecanismos de resolución de problemas en el uso del lenguaje. En este sentido, los trabajos de Dörnyei y sus colaboradores (Dörnyei y Scott, 1997; Dörnyei y Kormos, 1998) nos indican que los hablantes pueden recurrir a tres tipos de mecanismos cuando perciben que el mensaje a producir re- 
quiere más tiempo del que el sistema de producción o el contexto de comunicación le permiten:

a) reducir o abandonar el mensaje previamente planificado, tratando de evitar un discurso demasiado titubeante a consecuencia de los procesos de planificación subyacentes,

b) emplear algún mecanismo de codificación alternativo que resulte más rápido que codificar el mensaje preverbal, o

c) aplicar mecanismos de dilación (por ejemplo, introducir pausas, muletillas, repeticiones, etc.) que mantengan el canal de comunicación abierto mientras ganan tiempo y recursos para planificar y codificar la siguiente emisión.

Las dos primeras opciones demandan cierta cantidad de recursos cognitivos, aunque menos que la codificación del mensaje preverbal original, y pueden ir o no acompañadas de los mecanismos de dilación. Las estrategias para ganar tiempo no demandan procesamiento adicional cuando ya están automatizadas, aunque podrían resultar inadecuadas para mantener una comunicación fluida si dan lugar a un discurso titubeante y poco conexo. Incluso, una vez que el mensaje se ha verbalizado, el propio hablante puede darse cuenta de sus propios errores en la producción y repararlos inmediatamente.

Por otra parte, la premura de la comunicación en tiempo real requiere la automatización de los procesos lingüísticos básicos. En particular, en el uso de la L2 y en función del nivel de competencia lingüística del hablante, dichos procesos pueden demandar gran cantidad de recursos atencionales y de tiempo de procesamiento. Trabajos realizados recientemente desde la Psicología Cognitiva han puesto de manifiesto, por un lado, la importancia de los procesos de atención consciente en la adquisición y el desarrollo de cualquier interlengua (Schmidt, 1993) y, por otro, la limitación de recursos atencionales con que cuenta el sistema humano de procesamiento de la información (van Patten, 1990). Esto significa que, ante una tarea mediada por el lenguaje, el hablante ha de gestionar dichos recursos y priorizar ciertas demandas de procesamiento a expensas de otras (Anderson, 1995), por lo que algunos procesos pueden verse dificultados y dar lugar a una producción verbal menos fluida, menos precisa y/o menos compleja (Skehan, 1996).

En este marco, el estudio que presentamos trata de contribuir al conocimiento del nivel de competencia comunicativa de los escolares de ocho años, a fin de establecer un marco de referencia válido para intervenciones educativas orientadas a un mejor rendimiento en el área de la lengua oral. En concreto, intentamos conocer cómo varía dicha competencia en función del dominio del código empleado por el hablante: primera lengua vs segunda lengua. El trabajo se centra en el análisis del discurso oral producido por los escolares en ambas lenguas, tratando de identificar las principales dificultades con que se encuentran ante la premura de la comunicación en tiempo real y el déficit de recursos lingüísticos, así como las estrategias que emplean para superar sus limitaciones comunicativas. Para ello, analizamos la capacidad comunicativa que pueden desarrollar los escolares que están siguiendo un programa de inmersión parcial en la L2 y que pertenecen al grupo psicolingüístico predominante. La atención a la variable del código constituye un elemento de especial interés en nuestro contexto escolar, dado que puede contribuir al seguimiento de uno de los modelos de tratamiento de las dos lenguas presentes en la escuela y en el entorno social.

Nuestra hipótesis de trabajo mantiene que el nivel de competencia comunicativa será significativamente más elevado en la primera lengua que en la segunda, puesto que el do- 
minio del código es superior y los procesos básicos están automatizados. Es decir, los hablantes producirán en la L1 un discurso más fluido, debido a la mayor rapidez y precisión en los procesos de acceso al léxico, un discurso más preciso en la construcción del marco sintáctico, por un mejor conocimiento de las reglas del código, y una organización más elaborada de las ideas, construyendo estructuras gramaticales más complejas.

\section{Método}

\section{Participantes}

En nuestro estudio participó una muestra seleccionada al azar de 20 alumnos y alumnas de edad media 8,2 años, cuya L1 es el castellano. Todos ellos han seguido el mismo programa de inmersión lingüística parcial desde los seis años: la L2 (gallego) es la lengua vehicular de dos materias del currículum educativo de Educación Primaria y es objeto de estudio en otra durante tres horas semanales. La exposición a la L2 fuera del entorno escolar consideramos que ha sido la misma para todos los estudiantes.

\section{Materiales}

Analizamos la narrativa generada a partir de la observación de una secuencia de cinco láminas de contenido semánticamente relacionado y que, una vez ordenadas, constituyeron el soporte visual para relatar oralmente una historieta construida a partir de las escenas recogidas en las mismas. Para ello, hemos utilizado dos series de láminas de la Wchesler Intelligent Scale para niños (W.I.S.C.). Cada serie contiene una superestructura narrativa clara, con marco, tema, argumento y resolución (Thorndyke, 1977). Los resultados de estudios previos han puesto de manifiesto que los niños conocen y utilizan el esquema de los cuentos ya desde los 5 años (Vieiro y García Madruga, 1997), y que la capacidad de construir historias no depende del código que utilice el hablante (Bigas, Clariana, Guasch, Luna, Milian y Ribas, 1994). Por lo tanto, consideramos que ambos aspectos (el soporte visual del contenido semántico de la historia y el conocimiento de la superestructura del cuento) podrían facilitar la producción oral de los alumnos. En el Anexo A recogemos un ejemplo representativo de los relatos producidos por los participantes.

\section{Diseño}

Realizamos un diseño simple intrasujeto. Los niveles de la variable independiente vienen determinados por la lengua utilizada por el hablante (primera lengua, segunda lengua). Como variables dependientes hemos definido tres grupos de medidas que nos permiten analizar los siguientes aspectos de la competencia comunicativa: los procesos subyacentes de acceso al léxico, la construcción del marco sintáctico correcto según el código en uso y la complejidad en la organización de las ideas articuladas por el hablante. Adoptamos el modelo de análisis del discurso propuesto por Van Dijk y Kintsch (1983), según el cual la proposición constituye la unidad básica de información que subyace al discurso superficial y que tiene significado pleno con un valor de verdad en el mundo real o imaginario. Cada 
proposición se corresponde con una idea simple y está constituida por un predicado (verbo, adjetivo o preposición) que expresa conceptos relativos a estados, acciones o eventos, y uno o más argumentos que expresan el agente, objeto, meta o recipiente del predicado.

Con respecto a los procesos de acceso al léxico, definimos variables que reflejan la capacidad para acceder a la unidad léxica adecuada a la intención comunicativa del hablante o, en su caso, reparar el error cometido (reformulaciones, sustituciones, falsos inicios, cambios de código), así como índices de la velocidad de acceso a la palabra seleccionada y de la capacidad para hacer frente a la comunicación en tiempo real manteniendo un flujo lingüístico continuado (repeticiones, muletillas, titubeos, pausas y disfluencias) (Foster y Skehan, 1996). Con relación a la precisión en la construcción del marco sintáctico de la proposición, medimos errores que el hablante comete en la construcción de la forma gramatical (falta de concordancia sintáctica y falta de concordancia morfosintáctica) y en la conexión local entre proposiciones adyacentes (falta de coherencia local). Por último, analizamos qué tipo de relación mantienen las ideas generadas, asumiendo que a través de la coordinación y de la subordinación podremos obtener un índice del grado de complejidad o de estructuración interna del discurso (Skehan, 1996). En la tabla 1 se describe cada una de las variables anteriormente referidas. Asimismo, se pueden ver algunos ejemplos en el relato incluido en el Anexo A.

\section{Procedimiento}

Agrupamos aleatoriamente por parejas a todos los alumnos presentes en los grupos-clase que participaron en el experimento, a fin de no alertar a los sujetos experimentales de que sólo se iban a analizar sus datos. Durante dos sesiones experimentales, separadas por un intervalo de una semana, registramos en grabaciones magnetofónicas cada una de las narraciones producidas por todos los alumnos (40 en total). Citamos individualmente a las parejas en una sala cercana al aula ordinaria, seleccionamos aleatoriamente a uno de sus componentes como sujeto experimental y se le indicó que fuese el primero de la díada en realizar la tarea experimental. Contrabalanceamos las dos condiciones experimentales, de manera que cada semana los participantes pasaban por una condición experimental diferente, empezando la mitad de las parejas por la condición L1 y la otra mitad por la condición L2. Utilizamos una secuencia de láminas diferente en cada sesión. Ninguno de los sujetos experimentales tenía dificultades de aprendizaje ni una historia diferente de aprendizaje de las lenguas en proceso de adquisición. La instrucción que dimos a los alumnos para realizar la tarea fue la siguiente:

Estas láminas recogen las escenas de un cuento. En primer lugar, debes observarlas atentamente. Luego, has de narrar oralmente el cuento a tu compañero/a.

Una vez efectuadas las grabaciones, utilizamos el siguiente sistema de codificación. En primer lugar, realizamos la transcripción literal de cada uno de los discursos producidos oralmente, manteniendo todos sus elementos y el orden en que fueron verbalizados. A continuación, dividimos cada texto en proposiciones, siguiendo la técnica de análisis microproposicional de Turner y Greene (1977). Representamos cada proposición entre paréntesis, escribiendo el predicado seguido de sus argumentos, separados por comas. Por ejemplo, la cláusula El perro persiguió al gato asustado contiene dos proposiciones: P1 (PERSEGUIR, PERRO, GATO), P2 (ESTAR, GATO, ASUSTADO). 
TABLA 1: Descripción de las variables dependientes.

\begin{tabular}{|c|c|c|}
\hline Variable & Descripción & Ejemplo \\
\hline \multicolumn{3}{|c|}{ I. VARIABLES RELACIONADAS CON EL ACCESO AL LÉXICO } \\
\hline Reformulación & $\begin{array}{l}\text { Proposición que, una vez codificada oralmente, es repetida } \\
\text { inmediatamente por el hablante, manteniendo el significado } \\
\text { original pero introduciendo alguna modificación en su sintaxis o } \\
\text { en su morfología. }\end{array}$ & $\begin{array}{l}\text { Habían niños en } \\
\text { casa. } \\
\text { Había unos niños en } \\
\text { casa. }\end{array}$ \\
\hline Sustitución & $\begin{array}{l}\text { Proposición en la que alguno de los elementos originales } \\
\text { (predicado o algún argumento) se vuelve a codificar oralmente } \\
\text { con una nueva unidad léxica. }\end{array}$ & $\begin{array}{l}\text { El perro... el gato } \\
\text { estaba comiendo. }\end{array}$ \\
\hline Falso inicio & $\begin{array}{l}\text { Proposición que empieza a codificarse oralmente pero que se } \\
\text { interrumpe sin concluir su emisión, abandonando la idea } \\
\text { original. }\end{array}$ & $\begin{array}{l}\text { Ella tiene cui... Ella } \\
\text { estaba escribiendo. }\end{array}$ \\
\hline $\begin{array}{l}\text { Cambio de } \\
\text { código }\end{array}$ & $\begin{array}{l}\text { Proposición en la que alguno de sus elementos (predicado o } \\
\text { argumento) se codifica en la lengua diferente a la que se está } \\
\text { utilizando para producir el mensaje. Es decir, buscar el } \\
\text { equivalente léxico en la L1 cuando se está produciendo un } \\
\text { mensaje en la L2, o viceversa. }\end{array}$ & $\begin{array}{l}\text { Vin un chico } \\
\text { chorando. } \\
\text { (Chico es el } \\
\text { equivalente castellano } \\
\text { de la salida léxica } \\
\text { gallega mozo). }\end{array}$ \\
\hline Repetición & $\begin{array}{l}\text { Proposición en la que alguno de sus elementos (predicado o } \\
\text { argumento) se repite inmediatamente después de haberlo } \\
\text { codificado, sin modificar la sintaxis o la morfología. }\end{array}$ & $\begin{array}{l}\text { Estaba estaba sentada } \\
\text { en la escalera. }\end{array}$ \\
\hline Muletillas & $\begin{array}{l}\text { Palabras carentes de significado y de función gramatical, pero } \\
\text { que mantienen abierto el canal de comunicación. }\end{array}$ & Pues...; Eh...; Um... \\
\hline $\begin{array}{l}\text { Pausas y } \\
\text { disfluencias }\end{array}$ & $\begin{array}{l}\text { Silencio de una duración igual o superior a } 1.0 \text { segundo dentro } \\
\text { del turno de palabra del hablante. }\end{array}$ & \\
\hline Titubeos & $\begin{array}{l}\text { Repetición de fonemas o sílabas una o más veces antes de que } \\
\text { el hablante codifique oralmente la palabra completa. }\end{array}$ & $\ldots$ co...cop $\ldots$ copiaba \\
\hline \multicolumn{3}{|c|}{ II. VARIABLES RELACIONADAS CON LA PRECISIÓN SINTÁCTICA } \\
\hline $\begin{array}{l}\text { Falta de } \\
\text { coherencia } \\
\text { local }\end{array}$ & $\begin{array}{l}\text { Ausencia de proposiciones o de elementos proposicionales que } \\
\text { sirvan de puente de continuidad sintáctica, temporal o temática } \\
\text { entre proposiciones adyacentes mencionadas explícitamente en } \\
\text { el discurso. }\end{array}$ & $\begin{array}{l}\text { Ayer fui al cine y veré } \\
\text { una película de } \\
\text { ciencia-ficción. }\end{array}$ \\
\hline $\begin{array}{l}\text { Falta de } \\
\text { concordancia } \\
\text { sintáctica }\end{array}$ & $\begin{array}{l}\text { Proposición construida incorrectamente según las reglas del } \\
\text { código en uso, debido a que el hablante añade, suprime o } \\
\text { intercambia el orden de algún elemento proposicional. }\end{array}$ & $\begin{array}{l}\text { Te se te escapó la } \\
\text { pelota. }\end{array}$ \\
\hline $\begin{array}{l}\text { Falta de } \\
\text { concordancia } \\
\text { morfosintáctica }\end{array}$ & $\begin{array}{l}\text { Falta de correspondencia de género y/o número entre palabras } \\
\text { contenido (nombre, verbo, adjetivo) de la proposición, o entre } \\
\text { una palabra contenido y una palabra funcional (p.e., artículo) } \\
\text { que le acompaña. }\end{array}$ & Este libro son mío. \\
\hline \multicolumn{3}{|c|}{ III. VARIABLES RELACIONADAS CON LA COMPLEJIDAD GRAMATICAL } \\
\hline $\begin{array}{l}\text { Cláusula } \\
\text { simple }\end{array}$ & $\begin{array}{l}\text { Enunciado con independencia sintáctica y semántica, constituido } \\
\text { por una o más proposiciones y que incluye, al menos, un } \\
\text { predicado nominal o verbal. }\end{array}$ & $\begin{array}{l}\text { Había nieve en la } \\
\text { montaña. }\end{array}$ \\
\hline $\begin{array}{l}\text { Cláusulas } \\
\text { coordinadas }\end{array}$ & $\begin{array}{l}\text { Enunciado constituido por cláusulas unidas mediante un nexo } \\
\text { de coordinación que las relaciona a igual nivel sintáctico y } \\
\text { semántico. }\end{array}$ & $\begin{array}{l}\text { Fue a la tienda } \mathbf{y} \\
\text { compró patatas. }\end{array}$ \\
\hline $\begin{array}{l}\text { Cláusulas } \\
\text { subordinadas }\end{array}$ & $\begin{array}{l}\text { Enunciado constituido por cláusulas relacionadas mediante un } \\
\text { nexo de subordinación, el cual las conecta jerárquicamente a } \\
\text { distinto nivel sintáctico y semántico. }\end{array}$ & $\begin{array}{l}\text { Envié la carta que } \\
\text { estaba en la mesa. }\end{array}$ \\
\hline
\end{tabular}


Este sistema de análisis y codificación del discurso nos ha permitido puntuar las variables dependientes de la manera que exponemos a continuación. Para cada proposición, asignamos un «1» a la presencia y un «0» a la ausencia de cada una de las variables siguientes: reformulación, sustitución, falso inicio, cambio de código, repetición, muletilla, pausas y disfluencias, titubeos, falta de coherencia local, falta de concordancia sintáctica y falta de concordancia morfosintáctica. A continuación, transformamos las puntuaciones directas en porcentajes del total de proposiciones. Además, del número total de cláusulas, calculamos las cláusulas simples, las cláusulas coordinadas y las cláusulas subordinadas. Dos jueces puntuaron cada variable analizada, alcanzando un acuerdo interjueces del .90 .

\section{Resultados}

En general, los resultados sólo confirman parcialmente nuestra hipótesis inicial. Así, aunque se han producido ciertos efectos en la dirección que habíamos previsto, esto es, una comunicación oral más fluida y más precisa cuando los hablantes utilizaban la primera lengua, sin embargo, a diferencia de lo que esperábamos, el grado de complejidad gramatical fue similar en ambos códigos.

Como se muestra en la tabla 2, los resultados de los análisis descriptivos indican que las medidas relacionadas con el acceso al léxico, salvo la variable muletillas, siguieron un patrón definido por medias más elevadas, o al menos similares, cuando los participantes utilizaron la segunda lengua para comunicarse. Para explorar la diferencia estadística entre ambas condiciones utilizamos la Prueba $\mathrm{T}$ para muestras relacionadas, con un intervalo de confianza del $95 \%$. Tal y como refleja la tabla 3 , los resultados de estos análisis mostraron un incremento significativo de cambios de código, repeticiones, titubeos y pausas y disfluencias en los relatos producidos en la segunda lengua. Sin embargo, las medias de reformulaciones, sustituciones, falsos inicios y muletillas no fueron significativamente diferentes entre ambas condiciones. Estos resultados indican que la comunicación es más repetitiva y titubeante en la L2, con una clara presencia de la lengua materna de los hablantes. Las diferencias se vinculan, fundamentalmente, con medidas ligadas a la velocidad de acceso al sistema léxico y con la falta del equivalente planificado en la L2.

En cuanto a las medidas relacionadas con el procesamiento sintáctico, los resultados de los análisis descriptivos muestran que se producen más imprecisiones en la construcción morfosintáctica de la L2 que de la L1, así como mayor número de incoherencias entre proposiciones adyacentes (véase la tabla 2). La comparación de las medias con la Prueba T para muestras relacionadas, con un nivel a de .05 , reveló un incremento estadísticamente significativo de faltas de concordancia morfosintáctica en las producciones orales en la L2, mientras que el porcentaje medio de proposiciones con errores de concordancia sintáctica y de faltas de coherencia local no fue significativamente diferente entre ambas condiciones (véase la tabla 3).

Con respecto al grado de complejidad de las construcciones gramaticales producidas en ambas condiciones experimentales, nuestra hipótesis no se ha visto confirmada. En la tabla 2 se puede observar que la media de cláusulas simples fue ligeramente superior cuando los hablantes utilizaron la L2 para comunicarse, mientras que esta relación se invierte a favor de la L1 con respecto a las relaciones de coordinación y de subordinación entre las ideas explícitas en los relatos. Al realizar los análisis inferenciales con la Prueba T para muestras 


\section{TABLA 2: Medias y desviaciones típicas de las medidas de acceso al léxico, precisión sintáctica y complejidad gramatical en función de la lengua utilizada por el hablante.}

\begin{tabular}{|l|c|c|c|c|c|}
\hline \multirow{2}{*}{ Lengua } & \multirow{2}{*}{$\mathbf{N}$} & \multicolumn{2}{c|}{ Primera Lengua } & \multicolumn{2}{c|}{ Segunda Lengua } \\
\cline { 3 - 6 } & & $\overline{\mathbf{x}}$ & $\mathbf{s}^{\mathbf{2}}$ & $\overline{\mathbf{x}}$ & $\mathbf{s}^{\mathbf{2}}$ \\
\hline \multicolumn{7}{|c|}{ ACCESO AL LÉXICO: } \\
\hline Reformulación & 20 & 1.49 & 1.75 & 1.72 & 1.98 \\
\hline Sustitución & 20 & .59 & 1.00 & 1.01 & 1.23 \\
\hline Falso inicio & 20 & 2.32 & 2.48 & 2.15 & 2.53 \\
\hline Cambio Código & 20 & 1.78 & 6.58 & 17.75 & 10.98 \\
\hline Repetición & 20 & 2.12 & 2.11 & 6.03 & 4.81 \\
\hline Muletillas & 20 & 3.48 & 5.06 & 1.81 & 2.79 \\
\hline Pausas/Disfluencias & 20 & 12.13 & 7.09 & 18.06 & 12.05 \\
\hline Titubeos & 20 & 5.63 & 5.11 & 8.97 & 5.58 \\
\hline \multicolumn{7}{|c|}{ PRECISIÓN SINTÁCTICA: } & & \\
\hline Falta coherencia local & 20 & .66 & 1.33 & 1.32 & 2.14 \\
\hline F.Concordancia sintáctica & 20 & 2.60 & 3.37 & 2.45 & 1.57 \\
\hline F.Concordancia morfosintáctica & 20 & .47 & 1.44 & 1.40 & 2.25 \\
\hline \multicolumn{7}{|c|}{ COMPLEIDAD GRAMATICAL: } \\
\hline Cláusulas Simples & 20 & 2.38 & 2.22 & 2.64 & 3.37 \\
\hline Cláusulas Coordinadas & 20 & 12.9 & 3.52 & 12.67 & 3.79 \\
\hline Cláusulas Subordinadas & 20 & 10.15 & 3.64 & 10.14 & 3.35 \\
\hline
\end{tabular}

relacionadas, con un nivel a de .05, comprobamos que entre ambas condiciones no había diferencias estadísticamente significativas de cláusulas simples, coordinadas y subordinadas. Por lo tanto, a diferencia de lo que habíamos hipotetizado, no se produjeron variaciones en la organización de las ideas explícitas en las producciones orales en función de que el hablante haya utilizado la L1 o la L2 para comunicarse, sin apenas variación en el grado de coordinación y de subordinación de las construcciones gramaticales en ambas lenguas.

\section{Discusión}

En el trabajo que presentamos hacemos una exploración inicial de la competencia comunicativa desarrollada en función del código lingüístico utilizado por el hablante: primera lengua vs segunda lengua. En concreto, nuestro estudio se centra en el análisis del discurso oral producido en las dos lenguas en proceso de adquisición por estudiantes del primer cur- 
TABLA 3: Valores t, grados de libertad y niveles de significación de la diferencia de medias con la prueba $T$ para muestras relacionadas.

\begin{tabular}{|l|c|c|c|}
\hline \multicolumn{1}{|c|}{ Prueba T L1 - L2 } & t & gl & $\begin{array}{c}\text { p } \\
\text { (bilateral) }\end{array}$ \\
\hline \multicolumn{3}{|c|}{ ACCSO AL LÉXICO: } \\
\hline Reformulación & -.384 & 19 & .705 \\
\hline Sustitución & -1.111 & 19 & .280 \\
\hline Falso inicio & .239 & 19 & .813 \\
\hline Cambio Código & -6.353 & 19 & .000 \\
\hline Repetición & -2.567 & 19 & .029 \\
\hline Muletillas & 1.295 & 19 & .203 \\
\hline Pausas/Disfluencias & -1.895 & 19 & .030 \\
\hline Titubeos & -1.893 & 19 & .034 \\
\hline \multicolumn{5}{|c|}{ PRECISIÓN SINTÁCTICA: } \\
\hline Falta coherencia local & -1.371 & 19 & .186 \\
\hline Falta Concordancia sintáctica & 1.262 & 19 & .222 \\
\hline Falta Concordancia morfosintáctica & -2.724 & 19 & .008 \\
\hline \multicolumn{5}{|c|}{ COMPLEJIDAD GRAMATICAL: } \\
\hline Cláusulas Simples & -.283 & 19 & .778 \\
\hline Cláusulas Coordinadas & .194 & 19 & .846 \\
\hline Cláusulas Subordinadas & .011 & 19 & .991 \\
\hline
\end{tabular}

so del segundo ciclo de Educación Primaria. Los resultados muestran diferencias significativas en la competencia comunicativa desplegada en ambas lenguas, si bien dichas variaciones no afectaron como esperábamos a todas las variables analizadas. La hipótesis inicial mantenía que el nivel de competencia comunicativa sería significativamente más elevado en la primera lengua que en la segunda, dando lugar a un discurso más fluido, debido a la mayor rapidez y precisión en los procesos de acceso al léxico, un discurso más preciso en la construcción del marco sintáctico y más complejo en la estructuración de las ideas. Sin embargo, los resultados sólo apoyan este planteamiento con respecto al acceso a las unidades léxicas planificadas y a la construcción morfosintáctica, en el sentido que exponemos a continuación.

Como ya hemos señalado, la premura de la comunicación en tiempo real puede imponer ciertas restricciones de procesamiento debido, fundamentalmente, a la capacidad limitada de recursos cognitivos con que cuenta el hablante. Así, mientras que los procesos básicos de acceso al léxico o de codificación fonológica no estén automatizados, estarán demandando recursos atencionales en detrimento de otros procesos implicados en la construcción del 
significado y la forma del mensaje previamente planificado, especialmente cuando el hablante se encuentre en una situación de competencia lingüística limitada. En tales circunstancias, puede desplegar determinadas estrategias o mecanismos que le ayuden a superar las dificultades que la comunicación oral le plantea. En este sentido, la variable repeticiones ha reflejado un efecto claro de la lengua, aumentando significativamente en las narraciones producidas en la L2. De acuerdo con el esquema de análisis propuesto de Dörnyei y Kormos (1998), dicho mecanismo constituye una vía para mantener abierto el canal de intercambio comunicativo mientras el hablante gana tiempo y recursos para planificar la siguiente emisión. Si además consideramos el hecho de que se ha producido un aumento considerable de titubeos y de pausas y disfluencias en los relatos producidos en la L2, índices de la velocidad de acceso a las unidades léxicas seleccionadas (Dörnyei y Kormos, 1998) y de los procesos de planificación y organización de la información que va a decir a continuación (Foster y Skehan, 1996), respectivamente, los resultados sugieren que, a diferencia de lo que ocurre en la L1, los procesos básicos de carácter léxico y fonológico no se encuentran todavía automatizados en la L2, dificultando una comunicación fluida. Si asumimos que la fluidez de la producción oral refleja la primacía del significado y la capacidad para afrontar la comunicación en tiempo real (Schmidt, 1990; Skehan, 1996), así como la efectividad de los procesos de planificación de un discurso continuo y efectivo, podemos calificar el discurso producido por los escolares en la L2 como menos fluido, más repetitivo y titubeante que el producido en la L1, lo cual estaría en consonancia con los hallazgos de estudios previos (McWhinney, 1992; Towell, Hawkins y Bazergui, 1996; Wiese, 1984), lo que sugiere la existencia de más dificultades en la codificación del mensaje planificado.

Otro aspecto relevante es la presencia frecuente de la L1, o lengua materna, en los procesos de verbalización en la L2, como pone de manifiesto el alto porcentaje de cambios de código que se produjeron en dicha condición. Efectuado un análisis cualitativo de las narrativas generadas, interpretamos el uso de los cambios de código como una estrategia comunicativa intencional, en ocasiones, y como el resultado de una competencia lingüística en la L2 aún deficiente, en otras, dando lugar a producciones mixtas en ambos códigos. Su aparición puede ser debida a que la comunicación en tiempo real obliga a una búsqueda rápida del equivalente en cualquier lengua, de manera que el uso predominante de la L1 favorece un acceso más rápido y preciso al almacén de palabras del hablante, o incluso, que el castellano sea el único equivalente que conoce el individuo, por lo que accede a él para no interrumpir el flujo comunicativo de la L2. La revisión de los relatos nos indica que dichos cambios de código se basaron, fundamentalmente, en relaciones de tipo semántico y no fonológico, es decir, los participantes sustituyeron elementos léxicos al margen de principios o semejanzas fonológicas. Todo esto nos sugiere que los alumnos aún son dominantes en el uso de la lengua materna.

Con respecto al grado de precisión en la construcción del marco sintáctico proposicional, bien en su forma gramatical (concordancia sintáctica y morfosintáctica), bien en su continuidad temática o funcional (coherencia local), los resultados de nuestro estudio revelan diferencias significativas entre la L1 y la L2 en relación con la concordancia morfosintáctica. El análisis cualitativo de las transcripciones indica que sólo algunas construcciones causaron dificultades a los participantes, lo que, desde nuestro punto de vista, debe de estar indicando potenciales problemas para recuperar los morfemas de ciertas entradas léxicas. García Albea, del Viso e Igoa (1989) mantienen que este tipo de errores, indicativos de una falta de correspondencia entre el género y/o número de los distintos elementos de la 
frase, refleja restricciones de tipo gramatical y no tanto de tipo semántico. En este sentido, consideramos que este hecho puede estar relacionado con el grado de separación entre los dos sistemas lingüísticos en proceso de adquisición, de forma que las marcas propias de las dos lenguas utilizadas llevan a error a los sujetos que aún no tienen perfectamente diferenciados ambos códigos. Por ejemplo, suelen causar confusión los nombres que cambian de género según el código en uso (entre otros, el árbol en castellano y $a$ árbore en gallego).

De igual modo, pensamos que la semejanza estructural entre ambas lenguas es la razón subyacente para que no haya diferencias en la concordancia sintáctica y en la organización de las construcciones gramaticales construidas en cada código, generando discursos igualmente coherentes y complejos en ambas condiciones.

En resumen, podemos concluir que la competencia comunicativa en la L2 es diferente de la producida en la L1 en, al menos, los siguientes aspectos. En primer lugar, el hablante tiende a utilizar más mecanismos de dilación cuando utiliza la L2, posiblemente porque, como señala Levelt (1995), algunos procesos de formulación y articulación no están automatizados y, dependiendo del nivel de competencia en la L2, enlentecen el procesamiento del lenguaje. Por ello, la automatización de los procesos básicos de codificación fonológica y gramatical puede desempeñar un papel crucial en la liberación de recursos atencionales a favor de demandas lingüísticas y cognitivas de nivel más alto. En segundo lugar, la L1 tiende a influir notablemente en los procesos de verbalización de la L2, apareciendo fenómenos de interacción en los procesos léxicos y morfológicos. Finalmente, hemos de destacar que la comunicación fue efectiva en ambos códigos, de manera que aunque el habla fue menos fluida y menos precisa en la L2, las dificultades del intercambio oral no han interrumpido o dificultado la transmisión de significados. Así, los alumnos no han abandonado el mensaje preverbal, sino que han recurrido a ciertas estrategias comunicativas o mecanismos dilatorios que le facilitasen la recuperación de las unidades léxicas seleccionadas y los procesos de codificación y planificación de las subsiguientes emisiones.

Por último, quisiéramos subrayar el carácter limitado de este estudio. Se ha desarrollado en un contexto sociolingüístico particular, con la participación de un número de estudiantes relativamente reducido (20), pertenecientes a un único nivel educativo. De ahí que estos resultados deban ser considerados con precaución a la espera de más investigación. En este sentido, sería interesante comprobar si se mantiene el patrón descrito ampliando la muestra a otros niveles educativos y a otras lenguas con más distancia estructural. Por otra parte, comprobamos que la L2 producida es gramatical, aunque perteneciente a una interlengua todavía incorrecta que requiere mayor desarrollo. Queda abierto el interrogante sobre si las imprecisiones encontradas se hubiesen producido bajo otras condiciones experimentales no sometidas a la premura de la comunicación oral, como ocurre en el lenguaje escrito.

\section{Referencias}

ANDERSOn, J. R. (1995): Learning and memory. New York: John Wiley.

Bigas, M., Clariana, M., Guasch, O., Luna, X., Milian, M., y Ribas, T. (1994): La lengua escrita en la escuela: el texto narrativo a los ocho años. Infancia y aprendizaje, 65, 79-101.

Chaudron, C. (1988): Second Language Classrooms: Research on Teaching and Learning. Cambridge: Cambridge University Press. 
DöRNYEI, Z. (1995): On the teachability of communication strategies. TESOL Quarterly, 29, 55-85.

DöRNYEI, Z. y SCOTT, M. (1997): Communication strategies in a second language: definitions and taxonomies. Language Learning, 47, 173-210.

DörnyeI, Z. y Kormos, J. (1998): Problem-solving mechanisms in L2 communication. Studies in Second Language Acquisition, 20, 349-385.

EsteAire, S. y ZANÓN, J. (1990): El diseño de unidades didácticas mediante tareas: principios y desarrollo. Comunicación, lenguaje y educación, 7-8, 55-90.

Foster, K. y SKehan, P. (1996): The influence of planning and task type on second language performance. Studies in Second Language Acquisition, 18-3, 299-323.

García Albea, J. E., del Viso, S. E. IgOA, J. M. (1989): Movement errors and levels of processing in sentence production. Journal of Psycholinguistic Research, 18, 145-161.

LEVELT, W. (1989): Speaking from intention to articulation. Cambridge MA: the MIT Press.

LEVELT, W. (1995): The ability to speak: from intentions to spoken words. European Review, 3, 13-23.

McWhinney, B. (1992): Learning and memory. New York: John Wiley.

Poulisse, N. (1994): Communication strategies in a second language. En The Encyclopedia of language and linguistics (vol. 2, pp.620-624). Oxford: Pergamon Press.

Schmidt, R. (1990): The role of consciousness in second language acquisition. Applied Linguistics, $11,17-46$.

Schmidt, R. (1993): Awareness and Second Language Acquisition. Annual Review of Applied Linguistics, 13, 206-226.

SKeHAN, P. (1996): A framework for the implementation of task-based instruction. Applied Linguistics, 17/1, 38-62.

SWAIN, M. (1985): Communicative competence: some roles of comprehensible input and comprehensible output in its development. En Gass y Madden (eds.), Input in Second Language Acquisition. Cambridge: Cambridge University Press.

Thorndyke, P. (1977): Cognitive structures in comprehension and memory of narrative discourse. Cognitive Psychology, 9, 77-110.

Towel, R., Hawkins, R. y BAzergui, N. (1996): The development of fluency in advanced learners of French. Applied Linguistics, 17, 84-119.

Turner, A. y Greene, E. (1977): The construction of a propositional text base. JSAS Catalog of Selected Documents in Psychology.

VAN DiJK, LT. A. y KinTSCH, W. (1983): Strategies of discourse comprehension. New York: Academic Press.

Van Patten, B. (1990): Attending to content and form in the input: an experiment in consciousness. Studies in Second Language Acquisition, 12, 287-301.

Vieiro, P. y García Madruga, M. (1997): An analysis of story comprehension through spoken and written summaries in school-age children. Reading and Writting, 9, 41-53.

WIESE, R. (1984): Language production in foreign and native language: same or different? En H. DEChert, D. Mohle y M. Raupach (eds.), Second language productions (pp. 50-68). Tubingen: Gybter Narr. 


\begin{abstract}
ANEXO A
Ejemplo de un relato narrado por uno de los participantes, traducido al castellano para facilitar su lectura. Asimismo, incluimos entre paréntesis la codificación correspondiente de las medidas realizadas.

Érase una vez un chico, un chico (repetición) (c. simple). Yo creo que (subordinación) estaba viendo un escaparate ___ (pausa) y (coordinación) encuentran (falta de concordancia morfosintáctica) un amigo que (subordinación) se llamaba Javier. Eh... pues (muletilla) le dice si vie (falso inicio) (subordinación) si juega con él si viene a jugar con él (reformulación) y di dice (titubeo) que (subordinación) no que no (repetición) puede porque (subordinación) tiene inintel (palabra ininteligible) y (coordinación) dice entonces él: bueno (muletilla) vamos a sentarnos. El que (subordinación) está viendo el escaparate es bueno malo (sustitución) y (coordinación) se sentaron y (coordinación) el malo hizo así (gesto) con el banco, lo echó para adelante y (coordinación) el chico, y Javier, se puso para... (falso inicio) se cayó y (coordinación) se puso todo esto la cabeza con pájaros Y unas niñas estaban estaban (repetición) en otro banco y (coordinación) vio (falta de concordancia morfosintáctica) como (subordinación) le pegaban al chico y (coordinación) le riñeron. Vino vino (repetición) la policía y (coordinación) dij dijeron (titubeo) que (subordinación) si volvía hacer (falta concordancia sintáctica) que le meterían en la cárcel Al final se lo volvió a hacer al al (titubeo) otro y (coordinación) lo metieron en los calabozos y después (falso inicio) y (coordinación) tenía que estar (subordinación) (reformulación) 5 años. Estuvo 5 años y (coordinación) después salió del calabozo y (coordinación) al final fueron amigos. Y subió a casa (falta de coherencia local).
\end{abstract}

Fecha de recepción: 6-9-99

Fecha de revisión: 18-9-99

Fecha de aceptación: 11-4-00 